\title{
ASSESSMENT OF RECENT GOCE-BASED GLOBAL GEOPOTENTIAL MODELS AND EGM2008 IN NIGER REPUBLIC
}

\author{
Salissou IBRAHIM YAHAYA (iD) ${ }^{*}$, Driss EL AZZAB ${ }^{2}$ \\ ${ }^{1,2}$ Georessources and Environment Laboratory, Faculty of Sciences and Techniques, \\ Sidi Mohamed Ben Abdallah University, Mail box: 2202, Imouzzer Road, Fez, Morocco \\ ${ }^{1}$ Department of Surveying, Institute of Technology (IUT), University of Zinder, Mail box: 656, Zinder, Niger
}

Received 18 February 2019; accepted 18 September 2019

\begin{abstract}
In this study, we assessed recent GOCE-based Global Geopotential Models (GGMs) and EGM2008 in Niger. The combined GGMs EIGEN_6C4, GECO and EGM2008 were evaluated up to their maximum degree and order (d/o) 2,190 to select the one for gravity database densification. The following pure satellite GGMs were assessed for the modelling of the long and medium wavelengths in geoid computation: GGM05G, ITU_GGC16, EIGEN_6S4v2 and the fifth releases from direct (DIR5), space-wise (SPW5) and time-wise (TIM5) approaches. The GGMs are compared to terrestrial gravity data and geoid heights from GNSS/Levelling points before and after applying spectral enhancement method (SEM) by residual terrain model (RTM) for combined models and by RTM and the coefficients of selected combined GGM for pure satellite models. The agreements of combined GGMs with terrestrial gravity data and GNSS/Levelling points, in terms of root mean square (RMS) are about 4.88 to $5.02 \mathrm{mGal}$ and 0.14 to $0.16 \mathrm{~m}$, respectively. EIGEN_6C4 was selected as it showed the best performance in terms of geoid height differences and the probability of 3-sigma rule for gravity anomaly differences. At $\mathrm{d} / \mathrm{o}$ 200, DIR5 showed a good agreement with terrestrial gravity data $(5.04 \mathrm{mGal})$ and GNSS/Levelling points $(0.15 \mathrm{~m})$ after applying SEM, it was then retained. All GOCE-based models exhibited a good performance in long and medium wavelengths confirming the good recovery of the gravity field by the spatial gravity mission in these spectral bands.
\end{abstract}

Keywords: geopotential model, GOCE, EGM2008, geoid, gravity anomaly, GNSS/Levelling, Niger.

\section{Introduction}

The Global Geopotential Model (GGM) is a set of coefficients of a spherical harmonic expansion to a degree and order (d/o) of the Earth gravity field for global analysis. There are two categories of GGMs: pure satellite model and combined model which is obtained by merging spatial gravity measurements and terrestrial data eg. (Förste et al., 2016; Förste et al., 2014; Gatti \& Reguzzoni, 2017). The last three satellite gravity missions are CHAllenging Minisatellite Payload (CHAMP), Gravity Recovery and Climate Experiment (GRACE) and Gravity Field and Steady State Ocean Circulation Explorer (GOCE). Numerous GGMs were derived from the observations that represent the final scientific output for applications in oceanography, solid earth physics and geodesy. The GGMs are freely available on the website of the International Centre for Global Earth Models (ICGEM) (Barthelmes \& Köhler, 2016).

The expected accuracies from GOCE are of $1 \mathrm{mGal}$ and $1-2 \mathrm{~cm}$, respectively, for gravity anomalies and geoid, at spatial resolution of approximately $100 \mathrm{~km}$ half wavelength or d/o 200 of spherical harmonics (Drinkwater, Floberghagen, Haagmans, Muzi, \& Popescu, 2003). Up to date, five releases of GOCE-based GGMs have been developed, based on observation data in different time spans, by ESA's (European Space Agency) High Level Processing Facility (HPF). Measured data are processed through three different approaches: direct, time-wise and space-wise (Pail et al., 2011). The $5^{\text {th }}$ releases were based on the complete mission lifetime including the lower orbit data up to the re-entry of the satellite in November 2013 (Tocho \& Vergos, 2015). Besides the ESA's solutions, some models are based on the combination with other data sources, such as terrestrial gravity, satellite altimetry, or with CHAMP and GRACE eg. (Förste et al., 2016; Förste et al., 2014). GECO is an update of EGM2008 with GOCE data (Gilardoni, Reguzzoni, \& Sampietro, 2015).

In the remove-compute-restore (RCR) technique, the contribution of long and medium wavelengths of the

*Corresponding author. E-mail: salissou.ibrahimyahaya@usmba.ac.ma 
gravity field and the geoid, is computed from a pure satellite GGM (Omang \& Forsberg, 2000). The accuracy of the achieved geoid depends on that of the used GGM and it is sensitive to d/o ranging from 150 and 250 (Varga, Grgic, Bjelotomić, \& Bašić, 2018). The enhanced high-resolution combined GGMs by the residual terrain model (RTM) are also used for gravity database densification in areas with sparse data (Ulotu, 2009).

The gravity field quantities computed from a GGM are subject to the signal omission error comprising high frequency signals that cannot be represented by a truncated spherical harmonic series expansion (Hirt, Featherstone, \& Marti, 2010). In order to select the best GGM for gravity field modelling and geoid computation by RCR, several validation studies were carried out around the world, at local, regional or global scales eg. (Apeh, Moka, \& Uzodinma, 2018; Foroughi, Afrasteh, Ramouz, \& Safari, 2017; Godah \& Krynski, 2015; Yakubu, Ferreira, \& Asante, 2017). The quantities derived from GGMs are compared to terrestrial gravity anomalies, deflection of the vertical, existing geoids, GNSS/Levelling data and those from other GGMs. Some studies are based on direct comparison by simple differences between GGMs and terrestrial data at different d/o of truncation (Yakubu et al., 2017). Statistical measures such as standard deviation $(\sigma)$ and root mean square (RMS) of differences are analysed as function of d/o of truncation. Due to different spectral content between GGMs and terrestrial data, low-pass filters are commonly used to make the data comparable, such as Gaussian, average and inverse distance filters (Godah \& Krynski, 2015; Yakubu et al., 2017). However, the lowpass filters are more suitable for densely distributed or gridded data. The spectral enhancement is also used, the GGMs are completed with spherical harmonic coefficients of high-resolution combined GGMs such as EGM2008, and RTM (Hirt, Gruber, \& Featherstone, 2011). The RTM technique is capable of modelling major parts of highresolution GGM omission error and it can improve geoid determination in mountainous area (Hirt et al., 2010). The global assessment results are available on ICGEM website (Barthelmes \& Köhler, 2016). Several validation studies showed high performances and improvements worldwide of GOCE-based GGMs in medium and long wavelengths (El Brirchi \& El Azzab, 2011; Godah \& Krynski, 2015; Hirt et al., 2011; Yakubu et al., 2017). Also, the latest releases of GOCE-based GGMs derived from large time span measurements showed better performances (Tocho \& Vergos, 2015).

Up to date, there is no dedicated studies to assess the GGMs in general and GOCE-based ones in Niger. Some studies were done on the entire African continent and neighbouring countries such as Algeria, without a particular emphasis on Niger (Abd-Elmotaal, 2015; Benahmed Daho, 2010; Merry, 2009). This could be due to the inaccessibility or unavailability of sufficient validation data, including GNSS/Levelling points. The Niger Republic does not yet have a gravimetric geoid model and the gravity data are not densely distributed. The efforts are ongoing to compute the first geoid model in Niger. After the assessment of digital elevation models, the most suitable was used to compute high-resolution RTM effects for gravity field modelling and geoid computation (Ibrahim Yahaya \& El Azzab, 2018, 2019).

The main objective of this study is to select the best pure satellite GGM for the computation of long and medium wavelengths contributions in geoid determination by RCR and the combined model for gravity database densification in Niger. We therefore assess the recent GOCE-based GGMs and EGM2008. The pure satellite GGMs under evaluation are the fifth releases $\mathrm{GO}_{-}$ CONS_GCF_2_SPW_R5 (SPW5) (Gatti \& Reguzzoni, 2017), GO_CONS_GCF_2_TIM_R5 (TIM5) (Brockmann et al., 2014) and GO_CONS_GCF_2_DIR_R5 (DIR5) (Bruinsma et al., 2013), we included ITU_GGC16 (Akyilmaz et al., 2016), EIGEN-6S4v2 (Förste et al., 2016) and GGM05G (Bettadpur et al., 2015). The combined GGMs are EGM2008 (Pavlis, Holmes, Kenyon, \& Factor, 2012), EIGEN-6C4 (Förste et al., 2014) and GECO (Gilardoni et al., 2015). The GGMs are compared to terrestrial gravity data and GNSS/Levelling points before and after applying the spectral enhancement method by RTM, and by RTM and coefficients of combined GGMs, respectively, to combined GGMs and pure satellite ones. The differences are analysed by statistical measures.

\section{Materials and methods}

\subsection{Theory}

The geoid undulation $N_{G G M}$ and the gravity anomalies $\Delta g_{G G M}$ from a GGM are computed, respectively, using the expressions (Hofmann-Wellenhof \& Moritz, 2005):

$$
\begin{aligned}
& N_{G G M}=\frac{G M}{r \gamma_{0}} \times \\
& \sum_{n=2}^{+\infty}\left(\frac{R}{r}\right)^{n} \sum_{m=0}^{n}\left(\bar{C}_{n m} \cos m \lambda+\bar{S}_{n m} \sin m \lambda\right) \bar{P}_{n m}(\cos \theta)
\end{aligned}
$$

and

$$
\begin{aligned}
& \Delta g_{G G M}=\frac{G M}{r^{2}} \times \\
& \sum_{n=2}^{+\infty}(n-1)\left(\frac{R}{r}\right)^{n} \sum_{m=0}^{n}\left(\bar{C}_{n m} \cos m \lambda+\bar{S}_{n m} \sin m \lambda\right) \bar{P}_{n m}(\cos \theta)
\end{aligned}
$$

with $\gamma_{0}$ the normal gravity on the reference ellipsoid, $r$, $\lambda, \theta$ geocentric radius, longitude and colatitude of computation point, respectively. $R$ is the reference radius, $G M$ is the product of gravitational constant and the mass of the Earth, $n, m$ are the degree and order of spherical harmonic, $\bar{P}_{n m}$ are the fully normalised Legendre functions, $\bar{C}_{n m}$ and $\bar{S}_{n m}$ are fully normalised Stokes' coefficients of the disturbing potential. The infinite series is usually 
truncated at the maximum resolvable degree $n_{\text {max }}$ that is associated with a shortest resolvable wavelength at Earth's surface. In this study, we consider the terminology tailored in Šprlák, Gerlach, and Pettersen (2012): d/o 2 to 100 for low frequencies, 101 to 250 for medium wavelengths, 251 to 2,190 for high frequencies and the very-high frequencies for $\mathrm{d} / \mathrm{o}$ above 2,190 .

\subsection{Spectral enhancement method}

The main idea of the spectral enhancement method (SEM) is to fill the spectral gap between GGMs and terrestrial data (Hirt et al., 2011). For high-resolution GGMs such as EGM2008, omission error estimates sourced from RTM are used to recover the spectral band beyond $n_{\text {max }}=$ 2,190. A GGM (e.g. pure satellite model) under evaluation is evaluated up to degree $k_{1}$ and the spectral bands beyond this degree (from $k_{1}+1$ ) are recovered using a highresolution GGM and RTM omission error estimates. The omission error estimates are limited to the resolution of the digital elevation model used for RTM computation, for 3 arc-seconds resolution, it corresponds to d/o 216,000 (Hirt et al., 2011). The SEM is used for recovering various gravity field quantities, such as gravity anomalies, geoid undulations, height anomalies and deflections of the vertical (Hirt et al., 2010). The simplified principle of SEM is illustrated in Figure 1.

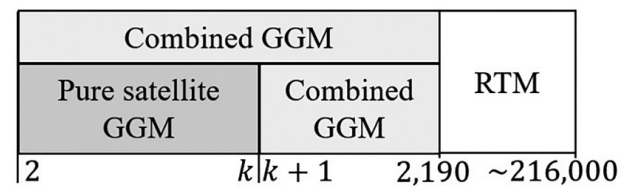

Figure 1. Simplified principle of Spectral Enhancement Method (SEM) (Hirt et al., 2011)

\subsection{Study area and terrestrial data}

The study area is located between $11^{\circ}$ to $24^{\circ}$ North and $0^{\circ}$ to $16^{\circ}$ East, and covers the Niger Republic and few other countries in the Central West Africa. The terrestrial gravity data cover the study area, they are provided by the International Gravimetric Bureau (BGI). We also retrieved gravity stations from ORSTOM (Office de Recherche Scientifique et Technique Outre Mer-France) technical report (Rechenmann, 1966). The quoted observation errors of simple Bouguer anomalies in Niger gravity database for the most unfavourable cases are $3.4 \mathrm{mgal}$ and $5.4 \mathrm{mgal}$ below and above the $16^{\text {th }}$ parallel, respectively. The merged data are 9,737 points of free air gravity anomalies $\Delta g_{F A}$, the values refer to GRS80 (Geodetic Reference System 1980) (Ibrahim Yahaya, El Brirchi, \& El Azzab, 2017b). Figure 2 presents the spatial distribution of gravity stations in the study area. The gravity database contains void areas in the southern of Algeria and Libya, and in the northern of Chad, Mali and Nigeria.

\subsection{GNSS/Levelling data}

The available 39 GNSS/Levelling points are located in the southwest part of Niger (Ibrahim Yahaya, El Brirchi, \& El Azzab, 2017a). They belong to the second order and the complementary levelling networks, with the accuracies of $5 \sqrt{S} \mathrm{~mm}$ and $5 \sqrt{S} \mathrm{~cm}$, respectively, where $S$ is the levelling distance in $\mathrm{km}$. They are also measured by differential GNSS using the static mode. We also retrieved 11 points of Nigerian geodetic network that are located in our study area from (Orupabo, Opuaji, \& Adekunle, 2014). The GNSS/Levelling points are plotted on Figure 3. The geoid undulation $N_{G P S}$ from GNSS/Levelling point is the difference between the ellipsoid height $h$ and the orthometric height $H$ :

$$
N_{G P S}=h-H \text {. }
$$

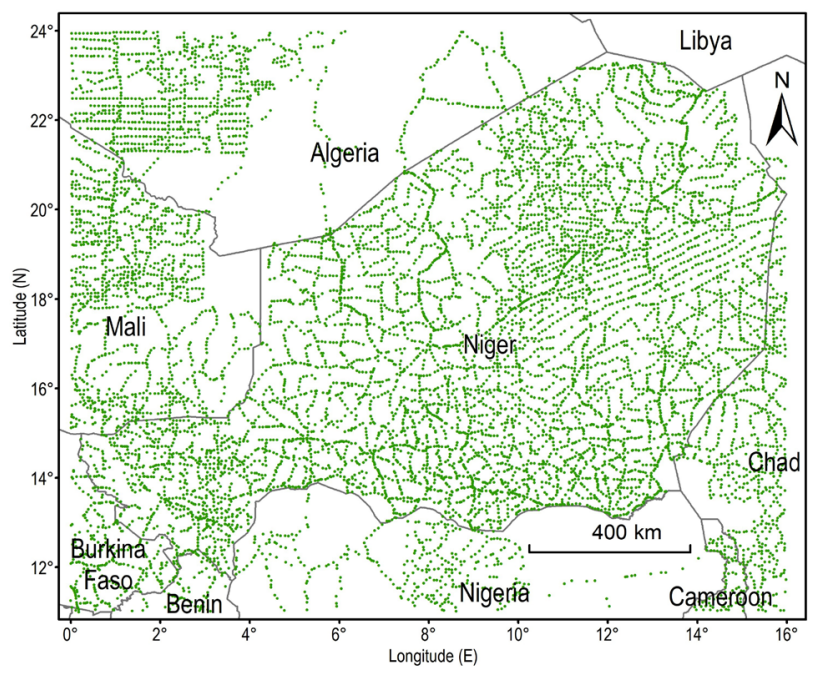

Figure 2. Spatial distribution of terrestrial gravity data

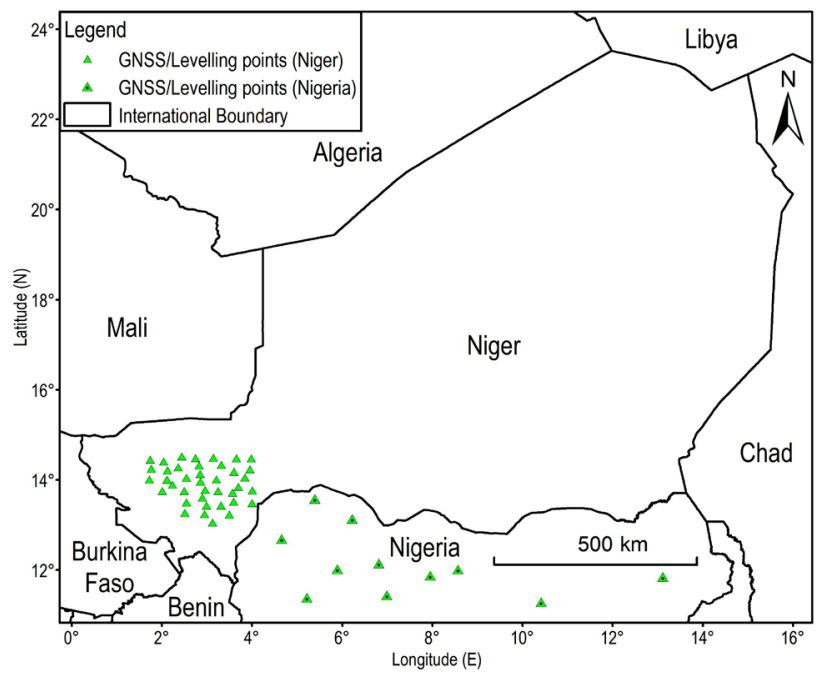

Figure 3. Spatial distribution of GNSS/Levelling points 


\subsection{Characteristics of GGMs under evaluation and computation of gravimetric quantities}

The GGMs were downloaded from the website of the ICGEM (Barthelmes \& Köhler, 2016), Table 1 presents their characteristics. The space-wise SPW5 and time-wise TIM5 solutions are GOCE-only GGMs, the direct approach solution DIR5 combines GRACE and LAGEOS data.

We computed gravity anomalies and geoid heights from all GGMs for d/o from 100 to 300 with five degrees of spectral interval. For gravity anomalies, 1.5' spaced grids were computed using the calculation service of ICGEM and values were extracted at each gravity station. The geoid undulations are computed at each GNSS/ Levelling point using GRAFIM Software (Janák \& Šprlák, 2006). The combined models are also evaluated up to their maximum d/o, 2,190. All quantities refer to GRS80.

Table 1. Models under evaluation

$\mathrm{S}=$ Satellite, $\mathrm{G}=$ Gravity, $\mathrm{A}=$ Altimetry

\begin{tabular}{|l|c|c|l|l|}
\hline \multicolumn{1}{|c|}{ Model } & Year & $\mathrm{d} / \mathrm{o}$ & \multicolumn{1}{c|}{ Data source } & \multicolumn{1}{c|}{ Reference } \\
\hline SPW5 & 2017 & 330 & S (GOCE) & $\begin{array}{l}\text { (Gatti \& } \\
\text { Reguzzoni, 2017) }\end{array}$ \\
\hline $\begin{array}{l}\text { ITU_ } \\
\text { GGC16 }\end{array}$ & 2016 & 280 & $\begin{array}{l}\text { S (GRACE, } \\
\text { GOCE) }\end{array}$ & $\begin{array}{l}\text { (Akyilmaz et al., } \\
\text { 2016) }\end{array}$ \\
\hline $\begin{array}{l}\text { EIGEN- } \\
\text { 6S4v2 }\end{array}$ & 2016 & 300 & $\begin{array}{l}\text { S (GOCE, } \\
\text { GRACE, } \\
\text { LAGEOS) }\end{array}$ & $\begin{array}{l}\text { (Förste et al., } \\
2016)\end{array}$ \\
\hline GECO & 2015 & 2190 & $\begin{array}{l}\text { S(GOCE), } \\
\text { EGM2008 }\end{array}$ & $\begin{array}{l}\text { (Gilardoni et al., } \\
\text { 2015) }\end{array}$ \\
\hline GGM05G & 2015 & 240 & $\begin{array}{l}\text { S (GRACE, } \\
\text { GOCE) }\end{array}$ & $\begin{array}{l}\text { (Bettadpur et al., } \\
\text { 2015) }\end{array}$ \\
\hline $\begin{array}{l}\text { EIGEN- } \\
\text { 6C4 }\end{array}$ & 2014 & 2190 & $\begin{array}{l}\text { S(GOCE, } \\
\text { GRACE, } \\
\text { LAGEOS), } \\
\text { G, A }\end{array}$ & $\begin{array}{l}\text { (Förste et al., } \\
\text { 2014) }\end{array}$ \\
\hline TIM5 & 2014 & 280 & $\begin{array}{l}\text { S (GOCE) } \\
\text { (Brockmann } \\
\text { et al., 2014) }\end{array}$ \\
\hline DIR5 & 2014 & 300 & $\begin{array}{l}\text { S (GOCE, } \\
\text { GRACE, } \\
\text { LAGEOS) }\end{array}$ & $\begin{array}{l}\text { (Bruinsma et al., } \\
\text { 2013) }\end{array}$ \\
\hline EGM2008 & 2008 & 2190 & $\begin{array}{l}\text { S(GRACE), } \\
\text { G, A }\end{array}$ & $\begin{array}{l}\text { (Pavlis et al., } \\
\text { 2012) }\end{array}$ \\
\hline
\end{tabular}

\subsection{Assessment of combined models}

The quantities $X_{\text {Comb }}^{2190}$ derived from combined GGMs up to their maximum $\mathrm{d} / \mathrm{o} 2,190$ are compared to those of terrestrial data and GNSS/Levelling points $X$ before and after applying SEM. Before the SEM, the difference $\delta X$ is given by:

$$
\delta X=X-X_{\text {Comb }}^{2190}
$$

where $X$ stands for gravity anomalies $\Delta g$ or geoid undulations $N$.

We used the grids of RTM effects derived from the reference topographic surface corresponding to about $9 \mathrm{~km}$ of spatial scale or d/o beyond 2,190 (Ibrahim Yahaya \&
El Azzab, 2018). The values of the indirect effect on geoid and the RTM gravity effect were extracted at each gravity station and GNSS/Levelling point. By applying the SEM using the RTM contributions $X_{R T M}$ to gravity anomalies or geoid, the differences are given by:

$$
\delta X=X-\left(X_{\text {Comb }}^{2190}+X_{R T M}\right) .
$$

The descriptive statistics of the differences are analysed in terms of maximum (Max), minimum (Min), mean $(\mu)$, standard deviation $(\sigma)$, root mean square (RMS) and histograms. We performed the Jarque-Bera normality tests at $5 \%$ and $10 \%$ of confidence levels on gravity anomaly differences. According to the 3-sigma rule in statistics, 99.7\% of the data values fall within three $\sigma$ of $\mu$ in either direction for normal distributions: $P[|\delta \Delta g-\mu| \leq 3 \sigma]=$ $99.7 \%$. We computed the probability for all GGMs. The best combined GGM will be used in the SEM for the pure satellite GGMs.

\subsection{Assessment of pure satellite GGMs}

The gravity anomalies or the geoid heights $X_{G G M}^{k}$ derived from pure satellite GGMs up to d/o $k=1,105,110 \ldots 300$ are compared to terrestrial free air anomalies and geometric geoid heights respectively. The differences are given by:

$$
\delta X=X-X_{G G M}^{k} \text {. }
$$

By applying the SEM, the differences are computed as follows:

$$
\delta X=X-\left(X_{G G M}^{k}+X_{C o m b}^{k+1 \text { to } 2190}+X_{R T M}\right),
$$

where $X_{\text {Comb }}^{k+1 \text { to } 2190}$ is the modelled quantity by the combined model from d/o $k+1$ to 2,190. The RMS values of the differences are analysed as function of $\mathrm{d} / \mathrm{o} k$.

\section{Results and discussions}

\subsection{Comparison of combined models}

\subsubsection{Gravity anomalies}

The histograms of gravity anomaly differences are plotted in Figure 4 for all combined GGMs before and after applying SEM, they are close to normal distributions from the same $\mu$ and $\sigma$ represented by the solid red lines. The Jarque-Bera normality tests showed that the differences of gravity anomaly do not follow normal distributions. The 3- $\sigma$ rule confirms this fact for all GGMs, the probabilities are less than $99 \%$ (Table 2). The distribution of gravity anomalies is not normal in general, and in our case, the existence of outliers in validation data could also explain the finding.

The statistics of the differences between the measured free-air anomalies and those modelled from the combined models, before and after SEM, are presented in Table 2. At $0.1 \mathrm{mGal}$ order, the models are similar before and after the application of SEM. EGM2008 has the smallest RMS, 4.88 $\mathrm{mGal}$, and GECO presents the greatest values, $5.02 \mathrm{mGal}$. The models show a slight increase in the range of 0.01 to 
Table 2. Statistics of differences between terrestrial gravity data and combined GGMs at d/o 2190 (in mGal)

\begin{tabular}{|l|l|c|c|c|c|c|c|c|}
\hline & \multicolumn{1}{|c|}{ Model } & MIN & MAX & $\mu$ & $\sigma$ & RMS & $P[|\delta \Delta g-\mu| \leq 3 \sigma]$ & No. \\
\hline \multirow{2}{*}{ Gravity anomalies } & $\Delta g_{\text {FA }}$ & -50.84 & 108.22 & -0.65 & 15.87 & 15.89 & - \\
\hline \multirow{3}{*}{$\begin{array}{l}\text { Differences before } \\
\text { SEM }\end{array}$} & EGM2008 & -36.70 & 45.90 & -1.28 & 4.71 & 4.88 & $98.57 \%$ \\
\cline { 2 - 9 } & EIGEN-6c4 & -37.15 & 48.01 & -1.32 & 4.78 & 4.96 & $98.77 \%$ \\
\cline { 2 - 9 } & GECO & -36.71 & 48.48 & -1.31 & 4.85 & 5.02 & $98.74 \%$ \\
\hline \multirow{3}{*}{$\begin{array}{l}\text { Differences } \\
\text { after SEM }\end{array}$} & $\Delta g_{\text {RTM }}$ & -12.34 & 24.65 & -0.20 & 1.13 & 1.14 & - \\
\cline { 2 - 9 } & EGM2008 & -42.65 & 45.69 & -1.08 & 4.77 & 4.89 & $98.73 \%$ \\
\cline { 2 - 9 } & EIGEN-6c4 & -41.60 & 47.79 & -1.12 & 4.85 & 4.98 & $98.80 \%$ \\
\cline { 2 - 9 } & GECO & -41.48 & 48.26 & -1.12 & 4.91 & 5.03 & $98.77 \%$ \\
\hline
\end{tabular}

$0.02 \mathrm{mGal}$ for RMS values after applying SEM. There is no improvement according to RMS values, other authors such as Šprlák, Gerlach, and Pettersen (2015) reported the contrary. On the other hand, we notice a decrease of $0.2 \mathrm{mGal}$ for the mean values of differences after the application of SEM, thus the reduction of the systematic bias. Also the probabilities $P[|\delta \Delta g-\mu| \leq 3 \sigma]$ increase from $98.57 \%$ to $98.73 \%, 98.77 \%$ to $98.80 \%$ and $98.74 \%$ to $98.77 \%$, respectively, for EGM2008, EIGEN_6C4 and GECO. EIGEN_6C4 showed the greatest probability values before and after SEM.

\subsubsection{Comparison of geoid heights}

The statistics of the difference between the geoid heights from GNSS/Levelling points and those modelled from the GGMs in Niger and northern Nigeria are presented in Table 3.
The first finding is that there is no improvement in geoid height in terms of $\sigma$ and RMS by RTM as it was reported in Šprlák et al. (2015), contrary to the results of other studies (Hirt et al., 2010; Kadlec, 2011). The RTM contributions to geoid heights are very low (less than $1 \mathrm{~cm}$ ) at the GNSS/Levelling points in our study area. EIGEN-6c4 and GECO have almost the same $\sigma$ and RMS values of differences. In Niger, they are slightly better than EGM2008, while in northern Nigeria EGM2008 performs better.

According to the results obtained with the gravity anomalies, EGM2008 is the candidate GGM for spectral enhancement and densification of the gravity database but it does not contain GOCE data. However, better precision is expected from the other two models in long and medium wavelengths because they contain GOCE data and EIGEN-6C4 showed the greatest probability according to
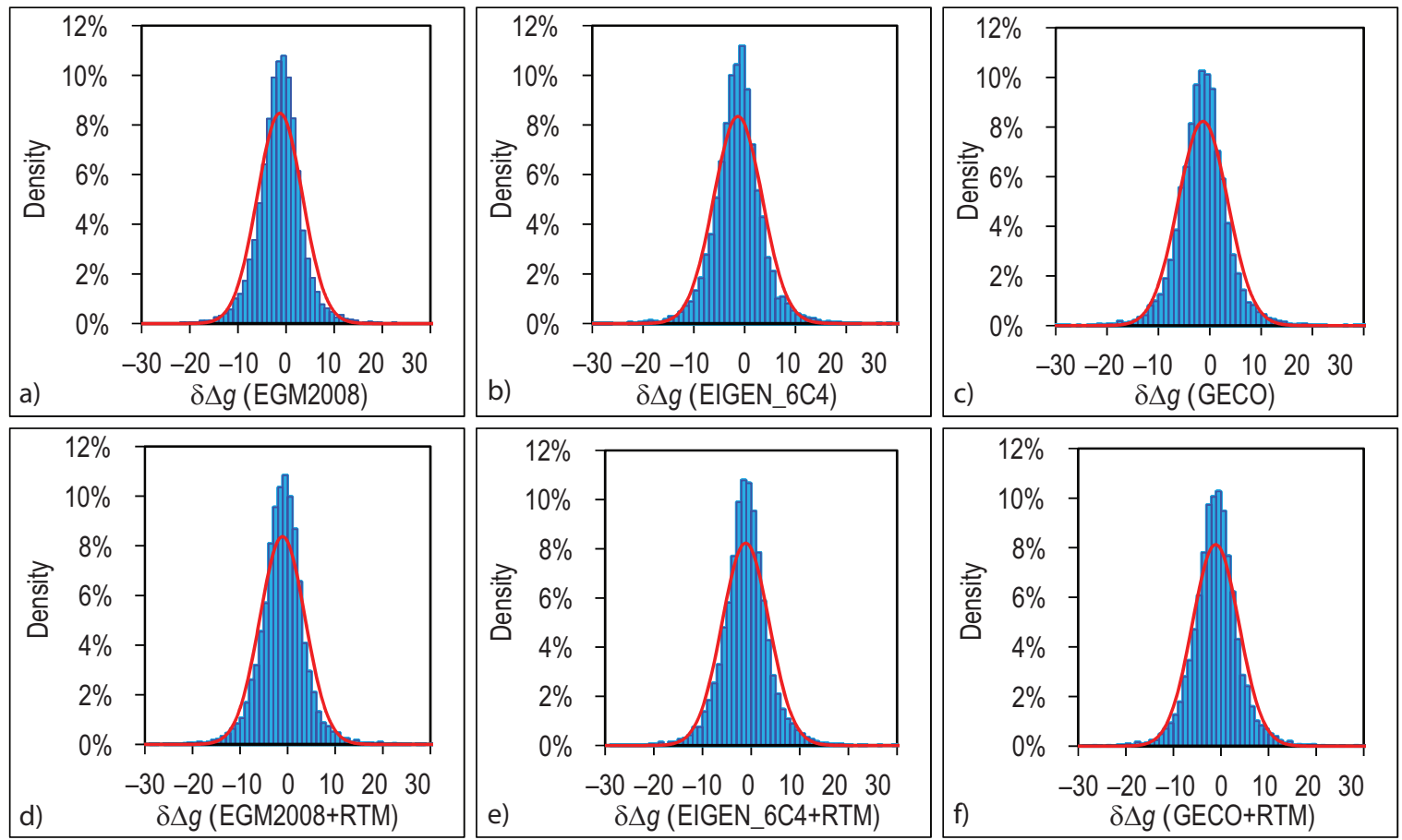

Figure 4. Histograms of gravity anomaly differences for combined GGMs up to d/o 2,190 and normal distributions from the same $\mu$ and $\sigma$ (Red). Before SEM: a - EGM2008; b - EIGEN_6C4; c - GECO. After SEM: d - EGM2008; e - EIGEN_6C4; f - GECO 
Table 3. Statistics of differences between GNSS/Levelling data and combined models at d/o 2190 (in metres)

\begin{tabular}{|c|c|c|c|c|c|c|c|c|}
\hline Area & SEM & Model & MIN & MAX & $\mu$ & $\sigma$ & RMS & No. \\
\hline \multirow{7}{*}{ Niger } & \multirow{3}{*}{ Before } & EGM2008 & -0.27 & 0.44 & 0.04 & 0.15 & 0.16 & \multirow{7}{*}{39} \\
\hline & & EIGEN-6c4 & -0.23 & 0.43 & 0.02 & 0.14 & 0.14 & \\
\hline & & GECO & -0.25 & 0.44 & 0.01 & 0.14 & 0.14 & \\
\hline & \multirow{4}{*}{ After } & $\mathrm{N}_{\mathrm{RTM}}$ & -0.003 & 0.005 & 0.001 & 0.002 & 0.002 & \\
\hline & & EGM2008 & -0.27 & 0.44 & 0.04 & 0.15 & 0.16 & \\
\hline & & EIGEN-6c4 & -0.23 & 0.43 & 0.02 & 0.14 & 0.14 & \\
\hline & & GECO & -0.25 & 0.44 & 0.02 & 0.14 & 0.14 & \\
\hline \multirow{7}{*}{ Nigeria } & \multirow{3}{*}{ Before } & EGM2008 & -1.05 & 1.95 & 0.46 & 0.83 & 0.92 & \multirow{7}{*}{11} \\
\hline & & EIGEN-6c4 & -1.10 & 1.89 & 0.47 & 0.85 & 0.94 & \\
\hline & & GECO & -1.10 & 1.88 & 0.48 & 0.86 & 0.95 & \\
\hline & \multirow{4}{*}{ After } & $\mathrm{N}_{\mathrm{RTM}}$ & -0.007 & 0.001 & -0.002 & 0.002 & 0.003 & \\
\hline & & EGM2008 & -1.06 & 1.95 & 0.46 & 0.83 & 0.92 & \\
\hline & & EIGEN-6c4 & -1.10 & 1.89 & 0.47 & 0.85 & 0.94 & \\
\hline & & GECO & -1.10 & 1.88 & 0.47 & 0.86 & 0.95 & \\
\hline
\end{tabular}

3-sigma rule. Also, for global assessments EIGEN-6C4 is presently used as a reference model (Barthelmes \& Köhler, 2016). We therefore retained EIGEN-6C4. In order to have an overview of the performance of these combined models in long and medium wavelengths, we included them in the comparison of "pure satellite" GGMs in the following section.

\subsection{Comparison of pure satellite GGMs with gravity anomalies}

\subsubsection{Before SEM}

The RMS values of differences for gravity anomalies as a function of truncation d/o prior to SEM are shown in Figure 5a. All models showed similar performance from d/o 100 to 215 compared to terrestrial data, RMS values decreased gradually with high d/o. From d/o 215, GGM05G stands out from other GGMs, with the largest gap up to its maximum degree, 240. Similarities between models continue for other GGMs up to d/o 230 where ITU_GGC16 and TIM5 stand out from other models.
From d/o 240, all pure satellite models become stable. ITU_GGC16 and TIM5 perform similarly up to d/o 280, also for EIGEN_6S4v2 and DIR5 up to d/o 300, and SPW5 is more accurate up to this $\mathrm{d} / \mathrm{o}$. Beyond $\mathrm{d} / \mathrm{o} 200$, the RMS values of differences for the combined models decrease progressively and similarly until d/o 300, GECO is less accurate than EGM2008 and EIGEN-6c4. EGM2008 appears similar to EIGEIN-6C4 and is better than other models. In these spectral bands, the combined models are generally better than the pure satellite GGMs due to the integration of terrestrial data that contain the full gravity spectrum.

\subsubsection{After SEM}

The RMS values for gravity anomalies differences as function of d/o after applying SEM are shown in Figure $5 b$. Up to d/o 180, all models have RMS curves coinciding with that of EIGEN-6C4 up to d/o 2,190 and enhanced by RTM, except GGM05G and EGM2008, which show poor performance compared to other models. The result of EGM2008 is justified by the fact that it does not contain GOCE data, on the other hand GGM05G combines
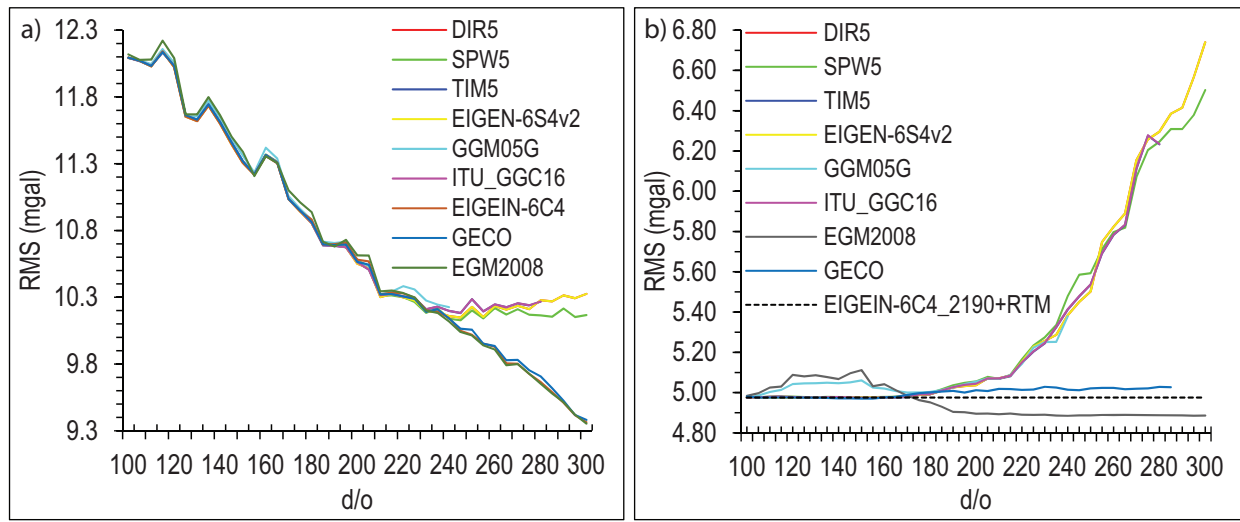

Figure 5. RMS of gravity anomaly differences as function of d/o of spherical harmonics: $a$ - before SEM; b - after SEM 
the data of ten (10) years of observations by GRACE and those of GOCE complete mission (Bettadpur et al., 2015). Beyond d/o 180, the RMS values for EGM2008 decrease gradually, those of GECO become stable, EGM2008 presents the best performance and EIGEN-6C4 is better than GECO. The RMS values of differences for pure satellite models are increasing exponentially. Table 4 shows the RMS values of differences for pure satellite GGMs up to d/o 180, 200, and 215 after SEM. The differences of RMS values between the models range from 0.01 to $0.02 \mathrm{mGal}$, at this level, DIR5 always has the smallest RMS value for the considered d/o. Abd-Elmotaal (2015) also found out that DIR5 better fits to the gravity field in Africa.

Table 4. RMS of gravity anomaly differences in $\mathrm{mGal}$ for pure satellite GGMs up to d/o 180, 200 and 215 after SEM

\begin{tabular}{|c|c|c|c|c|c|c|}
\hline d/o & DIR5 & SPW5 & TIM5 & $\begin{array}{c}\text { EIGEN- } \\
\text { 6S4v2 }\end{array}$ & GGM05G & $\begin{array}{c}\text { ITU_- } \\
\text { GGC16 }\end{array}$ \\
\hline 180 & 4.99 & 5.00 & 4.99 & 4.99 & 5.00 & 4.99 \\
\hline 200 & 5.04 & 5.06 & 5.04 & 5.04 & 5.05 & 5.04 \\
\hline 215 & 5.08 & 5.09 & 5.08 & 5.08 & 5.09 & 5.08 \\
\hline
\end{tabular}

\subsection{Comparison of pure satellite GGMs with GNSS/Levelling points}

\subsubsection{Before SEM}

RMS values of geoid height differences in Niger as function of $\mathrm{d} / \mathrm{o}$ prior to SEM are shown in Figure 6a. All models exhibit similar behaviour, except EGM2008 which deviates by about $2 \mathrm{~cm}$. Minimum values are reached at d/o 155, 180, and 230. From d/o 190 to 200, GECO and EIGEN-6C4 are better. From d/o 200 to 230, the pure satellite models, excepted GGM05G, are better than the combined models. The best performance of the pure satellite models is observed at d/o 230. At d/o 280, TIM5 and ITU_GGC16 are better, DIR5 and EIGEN_6S4 are better than SPW5 beyond this d/o. Up to d/o 300, EIGEN-6C4 is better than GECO and EGM2008.

The results in northern Nigeria are shown in Figure $6 \mathrm{~b}$. The models are also similar until d/o 200 at a cm level. At d/o 155, the minimum RMS value is reached for all models except for EGM2008, ITU_GGC16 gives the smallest value, $0.84 \mathrm{~m}$, while the largest value is shown by EGM2008. From d/o 200 to 300, the combined models show the best performance and EGM2008 is better than GECO and EIGEN-6C4. From d/o 200 to 240, DIR5 and ITU_GGC16 are better than other pure satellite models. From d/o 240 to 280, ITU_GGC16 is better among the pure satellite GGMs, also DIR5 and TIM5 are similar. Beyond d/o 280, DIR5 is better than EIGEN_6S4. SPW5 has the largest RMS values of difference between d/o 240 and 300. Overall, the differences of geoid heights between GNSS/Levelling points and GGMs in Niger are less than those obtained in northern Nigeria.

All pure satellite and combined models containing GOCE data showed a better performance compared to EGM2008 up to d/o ranging from 100 to 200. This confirms the improvement in the gravity field recovery in these spectral bands by GOCE gravity mission.
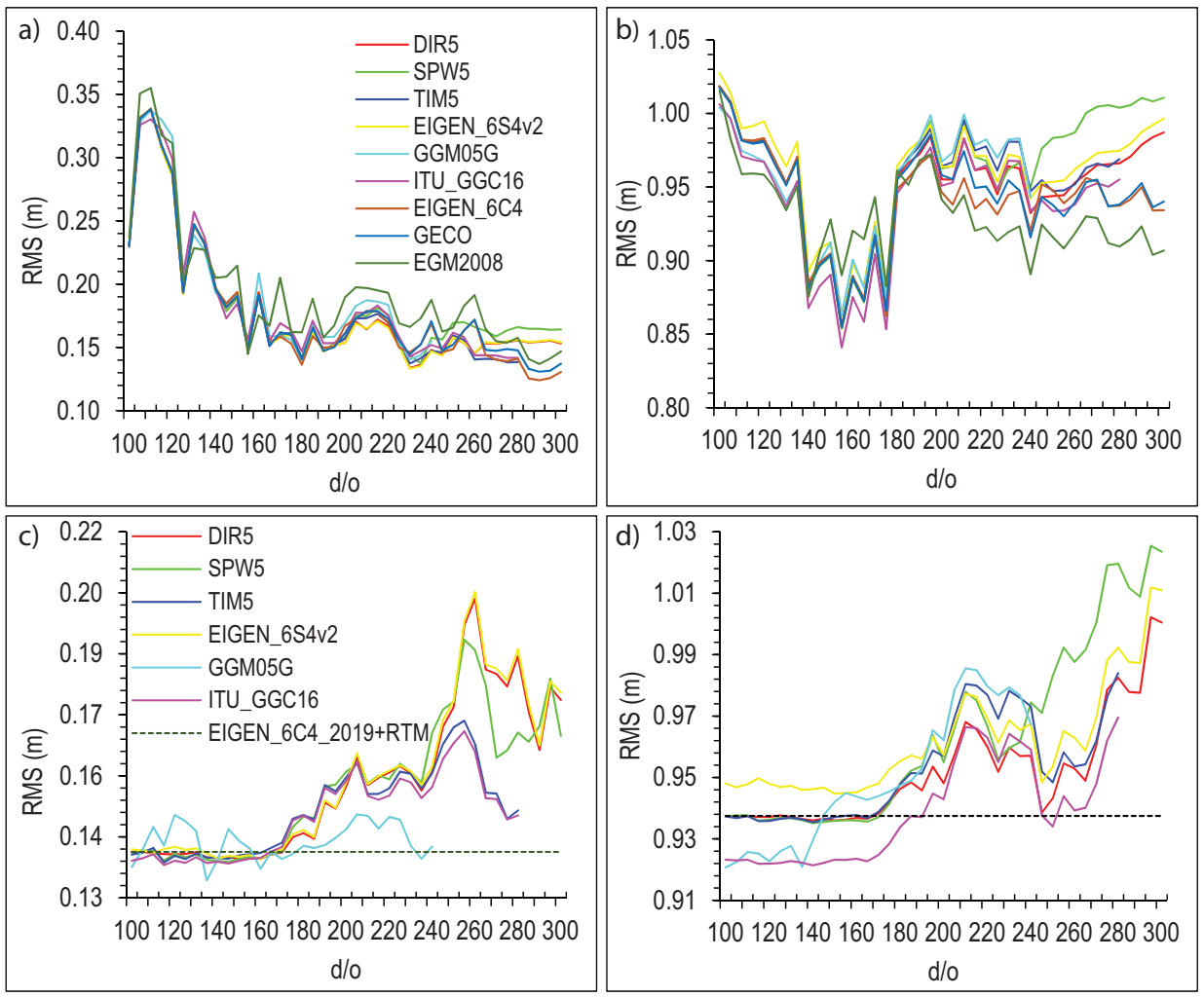

Figure 6. RMS of geoid height as function of d/o of spherical harmonics before SEM: a - in Niger; b - in Northern Nigeria; and after SEM: c - Niger; d - in Northern Nigeria 


\subsubsection{After SEM}

RMS values of geoid height differences as function of $d / o$ after SEM in Niger are shown in Figure 6c. All models behave similarly at the centimetre level until d/o 200 from which GGM05G presents the smallest RMS value. From d/o 170, RMS values become larger than that of EIGEN$6 \mathrm{C} 4$ enhanced by RTM. The results in northern Nigeria are shown in Figure 6d. They are similar to those in Niger, even though the differences are more important in this area. ITU_GGC16 present the smallest RMS values and EIGEN_6S4v2 the worst performance until d/o 200. Table 5 presents RMS values of geoid height differences for pure satellite GGMs at 200. In Niger, the smallest RMS value was observed with GGM05G $(0.14 \mathrm{~m})$ and the greatest with SPW5, the other models are similar. ITU_GGC16 showed the smallest RMS value (0.94 m), DIR5 and SPW5 are similar and the greatest value was observed with the remaining GGMs in northern Nigeria. In the two areas, ITU_GGC16 and DIR5 always perform better. As for gravity anomalies, DIR5 is suitable for modelling the long and medium wavelengths of the geoid in Niger.

Table 5. RMS of geoid height differences for pure satellite GGMs in metres at d/o 200 after SEM

\begin{tabular}{|l|c|c|c|c|c|c|}
\hline Area & DIR5 & SPW5 & TIM5 & $\begin{array}{c}\text { EIGEN_- } \\
\text { 6S4v2 }\end{array}$ & $\begin{array}{c}\text { GGM } \\
\text { 05G }\end{array}$ & $\begin{array}{c}\text { ITU_- } \\
\text { GGC16 }\end{array}$ \\
\hline Niger & 0.15 & 0.16 & 0.15 & 0.15 & 0.14 & 0.15 \\
\hline Nigeria & 0.95 & 0.95 & 0.96 & 0.96 & 0.96 & 0.94 \\
\hline
\end{tabular}

\section{Conclusions}

In this study, we assessed recent GOCE-based GGMs and EGM2008 in Niger Republic and surrounding areas. The quantities derived from combined GGMs EIGEN_6C4, GECO and EGM2008 evaluated up to their maximum d/o 2,190 were compared to free air gravity anomalies from terrestrial data and geoid undulations from GNSS/ Levelling points before and after applying SEM by RTM. We found out that the differences of gravity anomalies do no follow normal and the probability according to the 3 -sigma rule is near $99 \%$ for all models. In terms of RMS values of differences and at $0.1 \mathrm{mGal}$ order, EGM2008 is the best combined GGMs and GECO showed the worst performance before and after SEM. The combined GGMs showed a sensitivity of the gravity anomalies to the very short wavelengths modelled by RTM, the improvement was observed in terms of mean values of the differences and the probability according the 3 -sigma rule. EIGEIN_6C4 always gave the greatest probability value and further showed better performance than EGM2008 in long and medium wavelengths because it contains GOCE data. In the comparison of geoid undulations, the RMS of the differences in metres are: EGM2008 (0.16 and 0.92), EIGEN_6C4 (0.14 and 0.94) and GECO (0.14 and 0.95) respectively in Niger and northern Nigeria. EIGEN_6C4 showed better performance in Niger and EGM2008 in northern Nigeria. In GNSS/Levelling data areas, there is no improvement of geoid heights by RTM due to low lying terrain in general. We selected EIGEN_6C4 instead of EGM2008 for the SEM of pure satellite models and the densification of the gravity database in Niger.

The comparison of pure satellite GGMs to terrestrial gravity data, before SEM by EIGEN_6C4 and RTM, showed similar performance of all models, the values of RMS decreased with higher d/o until 250 where they became stable. Those of combined models continued decreasing. This finding confirmed the performance pure satellite GGMs only in long and medium wavelengths. After applying SEM, all GGMs behaved similarly to EIGEN_6C4 enhanced by RTM from d/o 100 to 180, except EGM2008 and GGM05G which showed the worst performance. At d/o 200, DIR5 showed the smallest value of RMS. Beyond d/o 180, the combined GGMs are better than the pure satellite ones.

The comparison between GGMs and GNSS/Levelling points before SEM showed also that GOCE-based are better than EGM2008 in long and medium wavelengths in Niger, and in northern Nigeria only up to d/o 200. After applying SEM, GGMs showed similar performance at 1 $\mathrm{cm}$ lever in Niger until d/o 180, where RMS values became larger than that the enhanced EIGEN_6C4 and GGM05G showed the best performance. In northern Nigeria ITU_ GGC16 presented the best performance. As with the gravity anomalies, DIR5 always showed small values of RMS at d/o 200, respectively $0.14 \mathrm{~m}$ and $0.95 \mathrm{~m}$ in Niger and northern Nigeria. We then selected DIR5 for modelling the long and medium wavelengths of the gravity anomalies and geoid in Niger.

This study confirmed the improvement in the gravity field recovery by GOCE spatial mission in the long and medium wavelengths. It is the first of its kind, carried out in Niger. As the main scientific contribution, we showed the performance of EGM2008 and recent GOCE-based models in this area of the African continent. This is also the result of efforts to collect sufficient data for this type of applications. The study provided an insight on the expected accuracy in GNSS levelling using the GGM-derived geoid models in the area of interest. However, efforts remain to be made in order to collect more GNSS/ levelling points.

\section{References}

Abd-Elmotaal, H. A. (2015). Validation of GOCE Models in Africa. Newton's Bulletin, (5), 149-162. Retrieved from http://www.isgeoid.polimi.it/Newton/Newton_5/11_Hussein_149_162.pdf

Akyilmaz, O., Ustun, A., Aydin, C., Arslan, N., Doganalp, S., Guney, C., Mercan, H., Uygur, S. O.; ,Uz, M., \& Yagci, O. (2016). ITU_GGC16 The combined global gravity field model including GRACE \& GOCE data up to degree and order 280. GFZ Data Services. https://doi.org/10.5880/icgem.2016.005

Apeh, O. I., Moka, E. C., \& Uzodinma, V. N. (2018). Evaluation of gravity data derived from global gravity field models using terrestrial gravity data in Enugu State, Nigeria. Journal of 
Geodetic Science, 8(1), 145-153.

https://doi.org/10.1515/jogs-2018-0015

Barthelmes, F., \& Köhler, W. (2016). International Centre for Global Earth Models (ICGEM). Journal of Geodesy, The Geodesists Handbook, 90(10), 907-1205.

https://doi.org/10.1007/s00190-016-0948-Z

Benahmed Daho, S. A. (2010). Assessment of the EGM2008 gravity field in Algeria using gravity and GPS/Levelling data. In International Association of Geodesy (IAG)-Gravity, Geoid and Earth Observation (pp. 459-466). Chania, Crete, Greece, 23-27 June 2008. Springer Berlin Heidelberg. https://doi.org/10.1007/978-3-642-10634-7_61

Bettadpur, S., Ries, J. C., Eanes, R., Nagel, P., Pie, N., Poole, S., Richter, T., \& Save, H. (2015). Evaluation of the GGM05 Mean Earth Gravity models. In Geophysical Research Abstracts-European Geosciences Union(EGU)2015-4153 (Vol. 17). Vienna, Austria.

Brockmann, J. M., Zehentner, N., Höck, E., Pail, R., Loth, I., Mayer-Gürr, T., \& Schuh, W.-D. (2014). EGM_TIM_RL05: An independent geoid with centimeter accuracy purely based on the GOCE mission. Geophysical Research Letters, 41(22), 8089-8099. https://doi.org/10.1002/2014GL061904

Bruinsma, S. L., Förste, C., Abrikosov, O., Marty, J.-C., Rio, M. H., Mulet, S., \& Bonvalot, S. (2013). The new ESA satellite-only gravity field model via the direct approach. Geophysical Research Letters, 40(14), 3607-3612. https://doi.org/10.1002/grl.50716

Drinkwater, M. R., Floberghagen, R., Haagmans, R., Muzi, D., \& Popescu, A. (2003). GOCE: ESA's first earth explorer core mission. In G. Beutler, M. R. Drinkwater, R. Rummel, \& R. Von Steiger (Eds.), Earth Gravity Field from Space - From Sensors to Earth Sciences (Vol. 17, pp. 419-432). Springer Netherlands. https://doi.org/10.1007/978-94-017-1333-7_36

El Brirchi, E. H., \& El Azzab, D. (2011). Improving geoid determination over Morocco area using Goce Level 2 Data. In 4th International GOCE User Workshop' (p. 6). Technical University of Munich, Germany.

Foroughi, I., Afrasteh, Y., Ramouz, S., \& Safari, A. (2017). Local evaluation of Earth Gravitational Models, case study: Iran. Geodesy and Cartography, 43(1), 1-13.

https://doi.org/10.3846/20296991.2017.1299839

Förste, C., Bruinsma, S. L. Abrikosov, O., Rudenko, S., Lemoine, J.-M., Marty, J.-C., Neumayer, K. H., \& Biancale, R. (2016). EIGEN-6S4: A time-variable satellite-only gravity field model to d/o 300 based on LAGEOS, GRACE and GOCE data from the collaboration of GFZ Potsdam and GRGS Toulouse. V. 2.0. GFZ Data Services. https://doi.org/10.5880/icgem.2016.008

Förste, C., Bruinsma, S. L., Abrikosov, O., Lemoine, J.-M., Marty, J. C., Flechtner, F., Balmino, G., Barthelmes, F., \& Biancale, R. (2014). EIGEN-6C4 The latest combined global gravity field model including GOCE data up to degree and order 2190 of GFZ Potsdam and GRGS Toulouse. GFZ Data Services. https://doi.org/10.5880/icgem.2015.1

Gatti, A., \& Reguzzoni, M. (2017). GOCE gravity field model by means of the space-wise approach (release R5). GFZ Data Services. https://doi.org/10.5880/icgem.2017.005

Gilardoni, M., Reguzzoni, M., \& Sampietro, D. (2015). GECO: a global gravity model by locally combining GOCE data and EGM2008. Studia Geophysica Et Geodaetica. https://doi.org/10.1007/s11200-015-1114-4

Godah, W., \& Krynski, J. (2015). Comparison of GGMs based on one year GOCE observations with the EGM08 and terrestrial data over the area of Sudan. International Journal of Applied Earth Observation and Geoinformation, 35, 128-135.

https://doi.org/10.1016/j.jag.2013.11.003
Hirt, C., Featherstone, W. E., \& Marti, U. (2010). Combining EGM2008 and SRTM/DTM2006.0 residual terrain model data to improve quasigeoid computations in mountainous areas devoid of gravity data. Journal of Geodesy, 84(9), 557567. https://doi.org/10.1007/s00190-010-0395-1

Hirt, C., Gruber, T., \& Featherstone, W. E. (2011). Evaluation of the first GOCE static gravity field models using terrestrial gravity, vertical deflections and EGM2008 quasigeoid heights. Journal of Geodesy, 85(10), 723-740. https://doi.org/10.1007/s00190-011-0482-y

Hofmann-Wellenhof, B., \& Moritz, H. (2005). Physical Geodesy. Vienna: Springer-Verlag. https://doi.org/10.1007/b139113

Ibrahim Yahaya, S., \& El Azzab, D. (2018). High-resolution residual terrain model and terrain corrections for gravity field modelling and geoid computation in Niger Republic. Geodesy and Cartography, 44(3), 89-99.

https://doi.org/10.3846/gac.2018.3787

Ibrahim Yahaya, S., \& El Azzab, D. (2019). Vertical accuracy assessment of global digital elevation models and validation of gravity database heights in Niger. International Journal of Remote Sensing, 1-20. https://doi.org/10.1080/01431161.2019.1607982

Ibrahim Yahaya, S., El Brirchi, E. H., \& El Azzab, D. (2017a). Impact of datum transformation on local variations of geometric geoid in Niger. Geodesy and Cartography, 43(4), 147-157. https://doi.org/10.3846/20296991.2017.1412615

Ibrahim Yahaya, S., El Brirchi, E. H., \& El Azzab, D. (2017b). Mise en place d'une base de données géographique pour le calcul du géoïde gravimétrique au Niger. In Congrès International MORGEO2017 sur les Technologies Géospatiales: Applications et Perspectives, 16-17 Mai 2017, Casablanca, Maroc.

Janák, J., \& Šprlák, M. (2006). A new software for gravity field modelling. Geodetic and Cartographic Horizon, (52), 1-8 (in Slovak).

Kadlec, M. (2011). Refining gravity field parameters by residual terrain modeling (Doctoral Thesis). Department of Mathematics, Faculty of Applied Sciences, University of West Bohemia, Pilsen, Czech Republic.

Merry, C. L. (2009). EGM2008 Evaluation for Africa. Newton's Bulletin, 4, 200-206. Retrieved from http://www.isgeoid.polimi.it/Newton/Newton_4/Report_EA10_Africa.pdf

Omang, O. C. D., \& Forsberg, R. (2000). How to handle topography in practical geoid determination: three examples. Journal of Geodesy, 74(6), 458-466.

https://doi.org/10.1007/s001900000107

Orupabo, S., Opuaji, T. A., \& Adekunle, I. A. (2014). 50-points data for deriving transformation parametrers of geodetic data in Nigeria. Indian Journal of Scientific Research and Technology, 2(1), 97-101.

Pail, R., Bruinsma, S., Migliaccio, F., Förste, C., Goiginger, H., Schuh, W.-D., ... Tscherning, C. C. (2011). First GOCE gravity field models derived by three different approaches. Journal of Geodesy, 85(11), 819-843. https://doi.org/10.1007/s00190-011-0467-x

Pavlis, N. K., Holmes, S. A., Kenyon, S. C., \& Factor, J. K. (2012). The development and evaluation of the Earth Gravitational Model 2008 (EGM2008). Journal of Geophysical Research, 117(B4), B04406. https://doi.org/10.1029/2011JB008916

Rechenmann, J. (1966). Catalogue des stations gravimétriques réoccupables en Afrique occidentale: réseau ORSTOM, mesures effectuées de 1953 à 1965. Cahier d'ORSTOM - Série Géophysique, 7, 195. Retrieved from http://www.documentation.ird.fr/hor/fdi:14966 
Šprlák, M., Gerlach, C., \& Pettersen, B. (2012). Validation of GOCE global gravity field models using terrestrial gravity data in Norway. Journal of Geodetic Science, 2(2), 134-143. https://doi.org/10.2478/v10156-011-0030-y

Šprlák, M., Gerlach, C., \& Pettersen, B. (2015). Validation of GOCE global gravitational field models in Norway. Newton's Bulletin, (5), 13-24. Retrieved from http://www.isgeoid.polimi.it/Newton/Newton_5/03_Sprlak_13_24.pdf

Tocho, C., \& Vergos, G. S. (2015). Assessment of different-generation GOCE-only and GOCE/GRACE Earth Global Gravity Models over Argentina using terrestrial gravity anomalies and GPS/Levelling data. Newton's Bulletin, (Special Issue 5), 105-126.
Ulotu, P. E. (2009). Geoid model of Tanzania from sparse and varying gravity data density by the KTH method. (PhD Dissertation). Department of Transport and Economics, Division of Geodesy, Royal Institute of Technology (KTH), 100 44 Stockholm, Sweden.

Varga, M., Grgic, M., Bjelotomić, O., \& Bašić, T. (2018). Investigation and comparison of RCR and LSMSA regional geoid modelling approaches. https://doi.org/10.13140/RG.2.2.35079.04008 Yakubu, C., Ferreira, V., \& Asante, C. (2017). Towards the selection of an optimal global geopotential model for the computation of the long-wavelength contribution: A case study of Ghana. Geosciences, 7(4), 113.

https://doi.org/10.3390/geosciences7040113 